Check for updates

Cite this: RSC Adv., 2018, 8, 36191

Received 3rd September 2018

Accepted 15th October 2018

DOI: $10.1039 / \mathrm{c} 8 \mathrm{ra07316c}$

rsc.li/rsc-advances

\section{A convenient separation method for di(2- ethylhexyl)phthalate by novel superparamagnetic molecularly imprinted polymers}

\begin{abstract}
Xinhua Yuan, (D)* Tiantian Liu, Lei Gao, Lu Xing, Yingying Zhu and Songjun Li
Through a surface molecular imprinting technique and coating superparamagnetic $\mathrm{Fe}_{3} \mathrm{O}_{4}$ nanoparticles with molecularly imprinted polymers (MIPs), a novel magnetic molecularly imprinted polymer (MMIP) was successfully fabricated for the convenient separation of di(2-ethylhexyl)phthalate (DEHP) with methacrylic acid as functional monomer, ethylene glycol dimethacrylate as cross linker and 2,2azobis(isobutyronitrile) as initiator. A magnetic non-molecularly imprinted polymer (MNIP) was also prepared for comparison purposes. The morphology structure and the magnetic properties of MMIP were characterized by transmission electron microscopy (TEM), Fourier transform infrared spectrometry (FT-IR), X-ray diffraction, vibrating sample magnetometry (VSM) and thermo gravimetric analysis (TGA). The adsorption properties of MMIP and MNIP were investigated by static and dynamic adsorption experiments. The results show that the diameter of the synthesized MMIP microspheres is about 300$500 \mathrm{~nm}$ with good dispersibility in solvent. The prepared MMIP shows superparamagnetic properties with the maximum saturation magnetic intensity of $43.97 \mathrm{emu}^{-1}$, and it can be conveniently separated using an external magnetic field. Compared with MNIP, MMIP has a higher adsorption capacity and better adsorption selectivity for DEHP, and the imprinting factor reaches 3.012. The regeneration adsorption experiment illuminates that the novel MMIP can be reused with good separation efficiency.
\end{abstract}

\section{Introduction}

Phthalates are a group of organic esters known as estrogens or endocrine disruptors, they are usually used as plasticizers to improve the flexibility and toughness of polyvinyl chloride. ${ }^{1,2}$ As a common phthalate, di(2-ethylhexyl)phthalate (DEHP) is widely used in many consumer products, such as building materials, medical devices, household products, clothes and cleaning materials. ${ }^{3}$ However, DEHP can easily leach from PVC and other products into the environment, and then transfer into water, air and food. When DEHP reaches a certain concentration, it will harm the health of human organisms, and cause wide adverse effects on the liver, reproductive tract, kidneys, lungs, fetus, and heart., ${ }^{4,5}$ Therefore, the development of new methods for determining and separating DEHP in diverse environmental matrices is highly significant.

Through molecular imprinting technique (MIT), molecularly imprinted polymers (MIPs) can be prepared by copolymerization of functional monomers and templates in the presence of cross-linkers and initiators, and the special recognition ability for a particular substance can be obtained. ${ }^{6}$ The threedimensional cavities complementary in shape, size and chemical functionality to those of templates are formed on the

School of Materials Science and Engineering, Jiangsu University, Zhenjiang, Jiangsu, 212013, China. E-mail: yuanxh@ujs.edu.cn; Fax: +86-0511-88780191 surface of prepared MIP, thereby can rebind the templates. MIPs have been widely used in solid phase extraction, ${ }^{7,8}$ chemical sensor, ${ }^{9,10}$ enzyme simulation and catalysis. ${ }^{11,12}$ MIPs are usually prepared by bulk polymerization with complicated process and deep recognition sites, which results in low binding affinity. In the process of preparation and application of MIPs, filtration or centrifugation is often needed, which is timeconsuming and difficult to be used widely in practical production. To solve the problems, novel magnetic surface imprinted polymers were reported recently, in which the binding sites were established on the surface of MIPs to facilitate the process of mass transfer and realize controllable movement by the external magnetic field. ${ }^{13-15}$ The magnetic molecular imprinting material can be used as an ideal and convenient separation material. However, there are few literatures of MMIPs for the separation of DEHP.

To realize convenient separation of DEHP with high efficiency, through surface molecular imprinting technique, a novel magnetic molecularly imprinted polymer (MMIP) was successfully synthesized by coating superparamagnetic $\mathrm{Fe}_{3} \mathrm{O}_{4}$ nanoparticles with MIPs and using methacrylic acid as functional monomer, ethylene glycol dimethacrylate as cross linker and 2,2-azobis(isobutyronitrile) as initiator. The morphology structure and the magnetic property of MMIP were analyzed by TEM, FT-IR, XRD, VSM and TGA, and the adsorption properties were also investigated. 


\section{Materials and methods}

\subsection{Chemicals}

DEHP, diallyl phthalate (DAP), dibutyl phthalate (DBP), tetraethoxysilane (TEOS), ferric chloride hexahydrate $\left(\mathrm{FeCl}_{3} \cdot 6 \mathrm{H}_{2} \mathrm{O}\right)$, ethylene glycol (EG), polyethylene glycol 2000 (PEG2000), sodiumacetate ( $\mathrm{NaAc}$ ), ammonia solution (28 wt\%), $\gamma$-(methacryloxypropyl) trimethoxy silane (MPS), ethylene glycol dimethylacrylamide (EGDMA), 2,2-azobis(isobutyronitrile) (AIBN), methacrylic acid (MAA), acetonitrile, ethanol, methanol, glacial acetic acid and toluene were all analytical reagent grade and purchased from Sinopharm Chemical Reagent Co., Ltd. (Shanghai, China).

\subsection{Preparation of MMIP}

First, $\mathrm{Fe}_{3} \mathrm{O}_{4}$ nanoparticles were synthesized by hydrothermal method. $\mathrm{FeCl}_{3} \cdot 6 \mathrm{H}_{2} \mathrm{O}(2.5 \mathrm{~g})$ was dissolved in $\mathrm{EG}(60 \mathrm{~mL})$ to form a clear solution by stirring. PEG2000 (2 g) and anhydrous NaAc $(7.2 \mathrm{~g})$ were added, and the mixture was stirred vigorously for an hour at $50{ }^{\circ} \mathrm{C}$. The mixture was transferred into a Teflon lined stainless-steel autoclave with $100 \mathrm{~mL}$ capacity, and the autoclave was heated to $200{ }^{\circ} \mathrm{C}$ and maintained for 8 hours. After that, it was cooled to room temperature naturally. The obtained $\mathrm{Fe}_{3} \mathrm{O}_{4}$ nanoclusters were washed with deionized water and ethanol for three times and separated from the supernatant by external magnetic field. Finally, the $\mathrm{Fe}_{3} \mathrm{O}_{4}$ nanoparticles were dried in vacuum oven at $50{ }^{\circ} \mathrm{C}$ for $6 \mathrm{~h}$.

Then, the $\mathrm{SiO}_{2}-\mathrm{Fe}_{3} \mathrm{O}_{4}$ microspheres were prepared by Stöber method. $0.2 \mathrm{~g} \mathrm{Fe}_{3} \mathrm{O}_{4}$ nanoparticles were dispersed by ultrasonic in $200 \mathrm{~mL}$ mixed solvent of ethanol and water $\left(\mathrm{v}_{\text {ethanol }}: \mathrm{v}_{\mathrm{water}}=\right.$ $4: 1)$, and $3 \mathrm{~mL} \mathrm{NH} \cdot \mathrm{H}_{2} \mathrm{O}(28 \mathrm{wt} \%)$ was rapidly added into the solution. After well-dispersed, $2 \mathrm{~mL}$ TEOS solvent was added drop by drop, and the reaction was proceeded at room temperature under mechanical stirring for 6 hours. The product was washed with ethanol and distilled water for three times and then dried in vacuum even at $50^{\circ} \mathrm{C}$ for $6 \mathrm{~h}$ to obtain $\mathrm{SiO}_{2}-\mathrm{Fe}_{3} \mathrm{O}_{4}$ microspheres.

The surface of $\mathrm{SiO}_{2}-\mathrm{Fe}_{3} \mathrm{O}_{4}$ particles was further modified with reactive $\mathrm{C}=\mathrm{C}$ bonds by reacting with $\gamma$-(methacryloxypropyl) trimethoxy silane. The obtained $\mathrm{SiO}_{2}-\mathrm{Fe}_{3} \mathrm{O}_{4}$ microspheres $(0.2 \mathrm{~g})$ were dispersed homogeneously in toluene (80 $\mathrm{mL}$ ) under ultrasonic condition. $6 \mathrm{~mL}$ MPS solvent was then added and reacted at $65^{\circ} \mathrm{C}$ with mechanical stirring for 24 hours under nitrogen protection. The obtained $\mathrm{MPS}-\mathrm{SiO}_{2}-\mathrm{Fe}_{3} \mathrm{O}_{4}$ microspheres were separated with the help of external magnetic field and washed repeatedly with ethanol and water. The MPS$\mathrm{SiO}_{2}-\mathrm{Fe}_{3} \mathrm{O}_{4}$ microspheres were also dried in vacuum even at $50{ }^{\circ} \mathrm{C}$ for $6 \mathrm{~h}$.

Finally, 0.25 mmol DEHP, 1 mmol MAA, $945 \mu \mathrm{L}$ EGDMA, $40 \mathrm{mg}$ AIBN and $0.1 \mathrm{~g}$ MPS- $\mathrm{SiO}_{2}-\mathrm{Fe}_{3} \mathrm{O}_{4}$ microspheres were dissolved in $80 \mathrm{~mL}$ acetonitrile with ultrasonic stirring for $15 \mathrm{~min}$. The mixture was polymerized at $65^{\circ} \mathrm{C}$ with mechanical stirring for 24 hours under the protection of nitrogen atmosphere. The product was washed with ethanol and distilled water for several times and collected by magnetic separation. The particles were eluted by the solution of methanol and acetic acid $(9: 1, \mathrm{v}: \mathrm{v})$ to remove the template molecules and dried in vacuum even at $50{ }^{\circ} \mathrm{C}$ for $12 \mathrm{~h}$ to obtain MMIP. To compare the separation performance, the magnetic non-molecularly imprinted polymer (MNIP) was also prepared in an identical manner but in the absence of template molecule.

\subsection{Characterization}

A JEM-2100 (HR) microscope was used to obtain transmission electron microscope (TEM) images of $\mathrm{Fe}_{3} \mathrm{O}_{4}$ nanoparticles, $\mathrm{SiO}_{2}-\mathrm{Fe}_{3} \mathrm{O}_{4}$ microspheres and MMIP microspheres. For TEM analyses, samples were prepared by placing one or two drops of nanoparticle solution onto the carbon-coated copper grid and drying it in air at room temperature.

Fourier transform infrared (FTIR) spectra were recorded using the FTIR spectrophotometer (Nicolet MX-1E, Thermo Fisher Scientific), and the scanning range is from $4000 \mathrm{~cm}^{-1}$ to $400 \mathrm{~cm}^{-1}$.

The identification of crystalline phase of the samples was performed by X-ray powder diffractometer with $\mathrm{Cu}$ KR radiation. The $2 \theta$ angles were from $10^{\circ}$ to $80^{\circ}$ at a rate $10^{\circ} \mathrm{min}^{-1}$.

Vibrating sample magnetometer (VSM) (Squid-VSM, USA) was used to test the magnetic properties of samples within $1 \mathrm{~T}$ at room temperature.

Ultraviolet visible spectrophotometer (UV-2800, SHIMADZU, Japan) was used to determine the concentration of DEHP in solution at room temperature.

The content and thermal stability of MPS- $\mathrm{SiO}_{2}-\mathrm{Fe}_{3} \mathrm{O}_{4}$ and MMIP were determined through thermo gravimetric analysis (TGA, STA449C, Germany) in the range from room temperature to $800{ }^{\circ} \mathrm{C}$ with a heating rate of $10{ }^{\circ} \mathrm{C} \mathrm{min}^{-1}$ under nitrogen atmosphere.

\subsection{Static adsorption experiments of MMIP and MNIP}

$20 \mathrm{mg}$ MMIP (or MNIP) was placed in the centrifuge tubes containing $2 \mathrm{~mL}$ ethanol solutions of DEHP with the concentrations from $20 \mathrm{mg} \mathrm{L}^{-1}$ to $200 \mathrm{mg} \mathrm{L}^{-1}$, and the mixture was shaken at room temperature for 12 hours. After the adsorption reached equilibrium, the adsorbent was separated from the solution by an external magnetic field. The concentration of DEHP in the supernatant solution was measured by UV spectrophotometer, and the detection wavelength of $225 \mathrm{~nm}$ was selected. The adsorption capacities $\left(Q_{\mathrm{e}}\right)$ of MMIP (or MNIP) for DEHP were calculated according to the following equation:

$$
Q_{\mathrm{e}}=\frac{\left(C_{0}-C_{\mathrm{e}}\right)}{m} V
$$

where $C_{0}\left(\mathrm{mg} \mathrm{L}^{-1}\right)$ is the initial concentration of DEHP solution, and $C_{\mathrm{e}}$ is the equilibrium concentration in supernatant solution $\left(\mathrm{mg} \mathrm{L}^{-1}\right) . V(\mathrm{~mL})$ is the volume of the DEHP ethanol solution, and $m(\mathrm{mg})$ is the mass of dry MMIP (or MNIP) that added to the solution.

\subsection{Dynamic adsorption experiment}

$20 \mathrm{mg}$ MMIP (or MNIP) was dispersed in $2 \mathrm{~mL}$ of $100 \mathrm{mg} \mathrm{L}^{-1}$ DEHP solution to investigate the kinetic adsorption properties. The mixture was shaken at room temperature for $10 \mathrm{~min}$, $20 \mathrm{~min}, 30 \mathrm{~min}, 40 \mathrm{~min}, 50 \mathrm{~min}, 1 \mathrm{~h}, 2 \mathrm{~h}$ and $3 \mathrm{~h}$, respectively. 
The microspheres were then separated by a magnet to determine the residual concentration of DEHP using UV spectrophotometer at $225 \mathrm{~nm}$. The adsorption capacity $Q$ was calculated according to formula (1).

\subsection{Selective adsorption experiment}

$20 \mathrm{mg}$ of dried MMIP and MNIP were placed in mixture solution of DEHP, DAP and DBP with initial individual concentrations of $100 \mathrm{mg} \mathrm{L}^{-1}$, and the mixture was respectively shaken at room temperature for $12 \mathrm{~h}$. The concentrations of DEHP, DAP and DBP in the supernatant solution was also measured by UV spectrophotometer.

\section{Results and discussion}

\subsection{Preparation of MMIP}

The synthesis process of MMIP was shown in Fig. 1. $\mathrm{Fe}_{3} \mathrm{O}_{4}$ magnetic nanoparticles were first synthesized through hydrothermal method by partial reduction of $\mathrm{FeCl}_{3}$ with $\mathrm{EG}$ as solvent, PEG as a surfactant against particle agglomeration and NaAc as electrostatic stabilization. ${ }^{16}$ A layer of $\mathrm{SiO}_{2}$ was subsequently coated on the surface of $\mathrm{Fe}_{3} \mathrm{O}_{4}$ particles to form $\mathrm{Fe}_{3} \mathrm{O}_{4} / \mathrm{SiO}_{2}$ coreshell particles by TEOS according to the Stöber method. ${ }^{17-19}$ The coating reaction is indispensable, because the silica shell is nontoxic and can provide good biocompatibility and hydrophilicity for $\mathrm{Fe}_{3} \mathrm{O}_{4}$ nanoparticles. The $\mathrm{SiO}_{2}$ shell can also effectively prevent the chemical degradation and aggregation of the $\mathrm{Fe}_{3} \mathrm{O}_{4}$ nanoparticles in a harsh liquid environment, and the silanol groups on the surface of silica can be further modified..$^{\mathbf{2 0}-22}$ Then, the silane coupling agent of MPS was used to modify the surface of silica to obtain reactive double bonds, which could interact with functional monomers and guide the formation of molecularly imprinted polymers on the surface of the magnetic matrix. Finally, the molecularly imprinted shell was coated on the surface of the magnetic substrate to obtain magnetic molecularly imprinted polymers. The polymer not only has specific recognition sites for template molecules, but also can be used for convenient separation by external magnetic field.

\subsection{TEM analysis}

The morphology structures of $\mathrm{Fe}_{3} \mathrm{O}_{4}, \mathrm{SiO}_{2}-\mathrm{Fe}_{3} \mathrm{O}_{4}$ and MMIP were observed by TEM (Fig. 2). Fig. 2(a) reveals that the

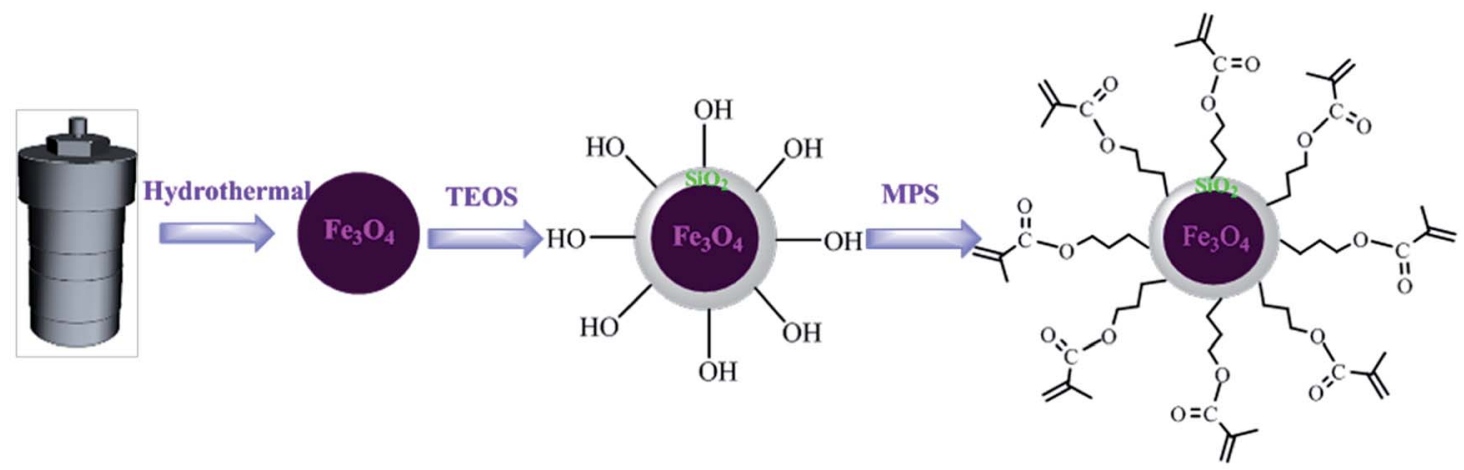

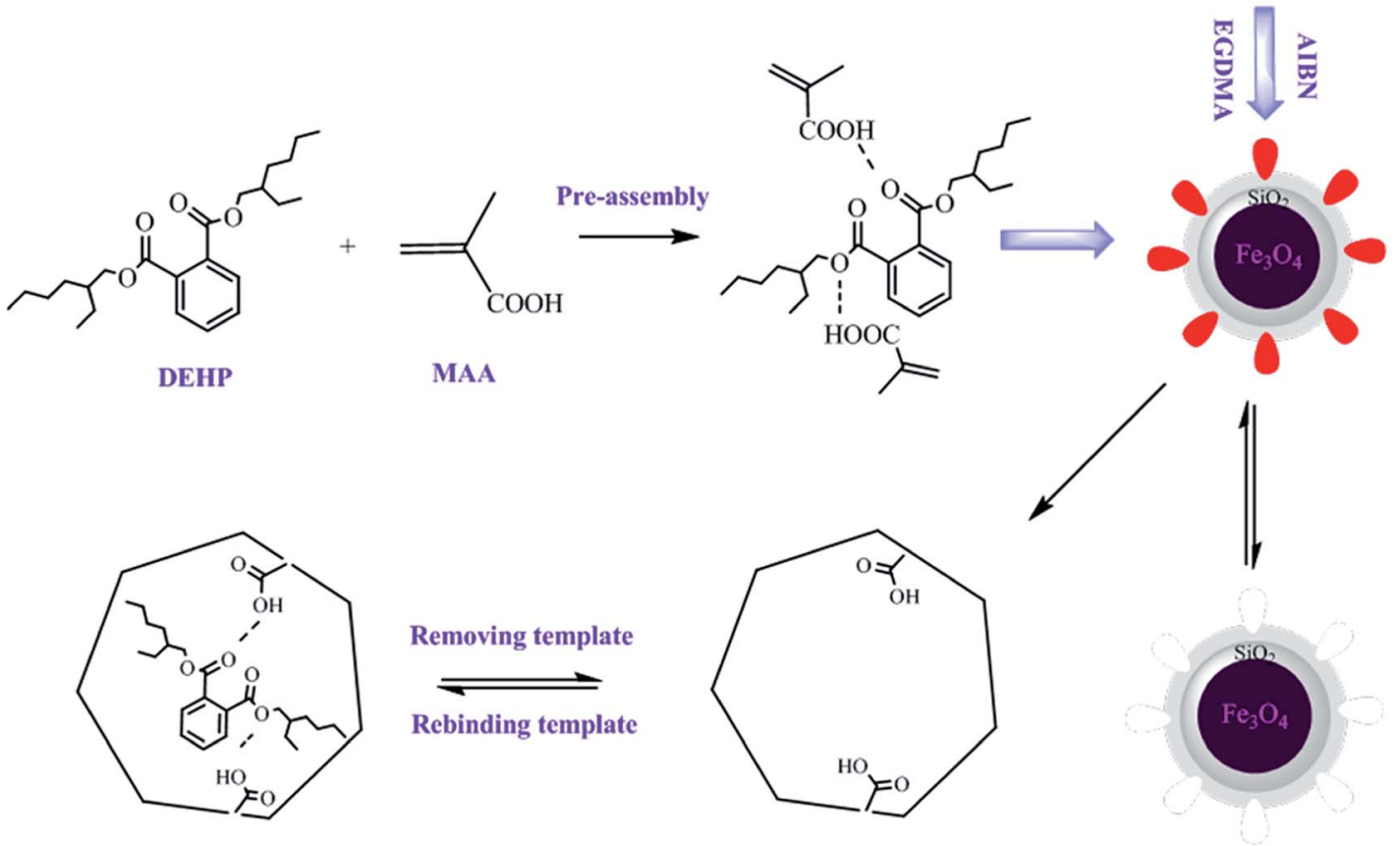

Fig. 1 Schematic diagram of MMIP preparation. 

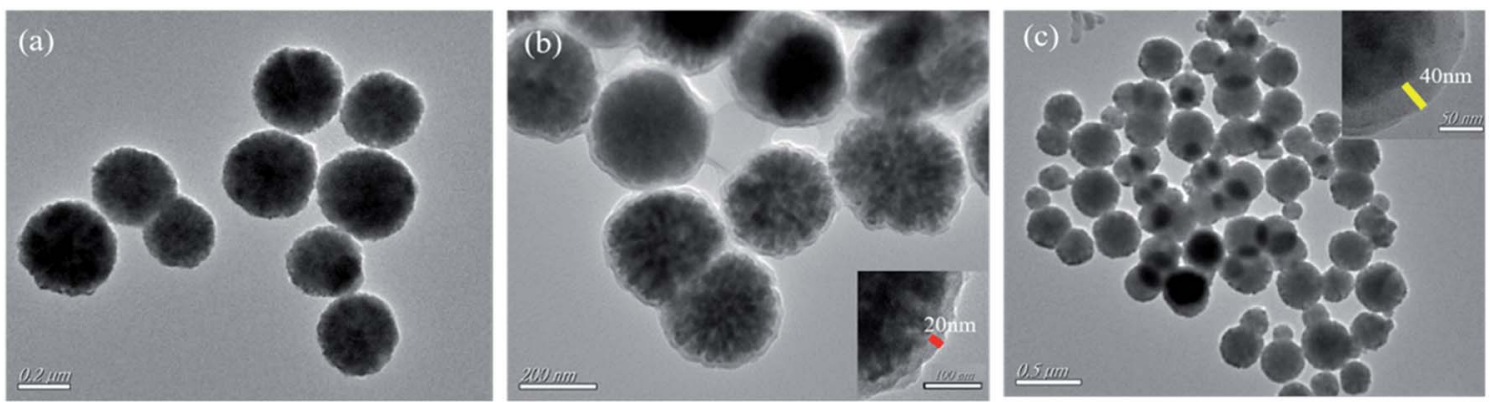

Fig. 2 TEM images of $\mathrm{Fe}_{3} \mathrm{O}_{4}$ (a), $\mathrm{SiO}_{2}-\mathrm{Fe}_{3} \mathrm{O}_{4}$ (b) and MMIP (c).

synthesized $\mathrm{Fe}_{3} \mathrm{O}_{4}$ nanoparticles have regular spherical shape with good dispersibility, and the diameter is in the range of $300-500 \mathrm{~nm}$. As shown in Fig. 2(b), $\mathrm{SiO}_{2}$ shell is coated on the surface of $\mathrm{Fe}_{3} \mathrm{O}_{4}$ and displays obvious core-shell structure, the thickness of the silica layer is about $20 \mathrm{~nm}$. Because the density of the silica shell is similar to that of molecularly imprinted shell, the molecularly imprinted shell cannot be distinguished clearly in Fig. 2(c). However, compared with Fig. 2(b), the thickness of the coating layer is further increased, which suggests the formation of imprinting shell.

\subsection{Infrared analysis}

Fig. 3 shows the infrared characterization of $\mathrm{Fe}_{3} \mathrm{O}_{4}, \mathrm{SiO}_{2}-\mathrm{Fe}_{3} \mathrm{O}_{4}$, MPS- $\mathrm{SiO}_{2}-\mathrm{Fe}_{3} \mathrm{O}_{4}$ and MMIP. The peak at $587 \mathrm{~cm}^{-1}$ in curve (a) is the typical vibration of the $\mathrm{Fe}-\mathrm{O}$, which also exists in curve (b), (c) and (d). The peaks at $1630 \mathrm{~cm}^{-1}$ in curve (a), (b), (c) and (d) are attributed to the bending vibration of $\mathrm{Fe}_{3} \mathrm{O}_{4}$ hydration water. The strong peaks at $1090 \mathrm{~cm}^{-1}, 798 \mathrm{~cm}^{-1}$ of curve (b) are attributed to the anti-stretching vibration of $\mathrm{Si}-\mathrm{O}-\mathrm{Si}$ and the symmetric stretching vibration of $\mathrm{Si}-\mathrm{O}$, respectively, indicating the formation of silica layer. The peaks at $2920 \mathrm{~cm}^{-1}$ and $2850 \mathrm{~cm}^{-1}$ in curve (c) correspond to the vibration of $\mathrm{C}-\mathrm{H}$, and the peak at $1640 \mathrm{~cm}^{-1}$ in curve (c) is the stretching vibration of $\mathrm{C}=\mathrm{C}$, which are attributed to the silica shell modified by MPS.

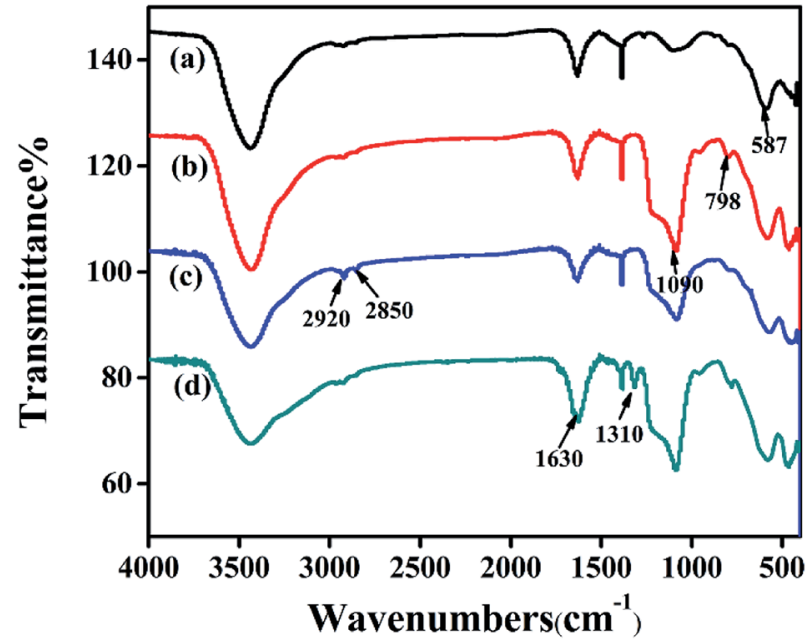

Fig. 3 FTIR spectra of $\mathrm{Fe}_{3} \mathrm{O}_{4}$ (a), $\mathrm{SiO}_{2}-\mathrm{Fe}_{3} \mathrm{O}_{4}$ (b), MPS $-\mathrm{SiO}_{2}-\mathrm{Fe}_{3} \mathrm{O}_{4}$ (c) and MMIP (d).
In curve (d), the peak at $1310 \mathrm{~cm}^{-1}$ is attributed to the stretching of $\mathrm{C}-\mathrm{O}$, which shows functional monomers are grafted on the surface of silica. Meanwhile, the peak at $1630 \mathrm{~cm}^{-1}$ is greatly enhanced, which suggests the formation of polymer shells on the surface of the $\mathrm{SiO}_{2}-\mathrm{Fe}_{3} \mathrm{O}_{4}$. All the results indicate the successful preparation of MMIP.

\subsection{TGA analysis}

The thermo gravimetric curves of MPS- $\mathrm{Fe}_{3} \mathrm{O}_{4}-\mathrm{SiO}_{2}$ and MMIP are shown in Fig. 4. Both the samples don't have obvious decline below the $200{ }^{\circ} \mathrm{C}$ expect for the slight weight loss of physically adsorbed water. From $200{ }^{\circ} \mathrm{C}$ to $800{ }^{\circ} \mathrm{C}$, the weight decreased $5.66 \%$ in curve (a), which is due to the degradation of silica layer coated on the surface of $\mathrm{Fe}_{3} \mathrm{O}_{4}$ and the MPS covered on the surface of $\mathrm{SiO}_{2}-\mathrm{Fe}_{3} \mathrm{O}_{4}$. However, the weight is decreased rapidly in curve (b), especially from $500{ }^{\circ} \mathrm{C}$ to $600{ }^{\circ} \mathrm{C}$, which is mainly due to the decomposition of the surface imprinting layer. The results also demonstrate the formation of imprinting layer, which is consistent with the TEM and FTIR analyses.

\subsection{XRD analysis}

XRD patterns of $\mathrm{Fe}_{3} \mathrm{O}_{4}, \mathrm{SiO}_{2}-\mathrm{Fe}_{3} \mathrm{O}_{4}$ and MMIP are shown in Fig. 5. Six characteristic peaks appear in the $2 \theta$ range of from 20

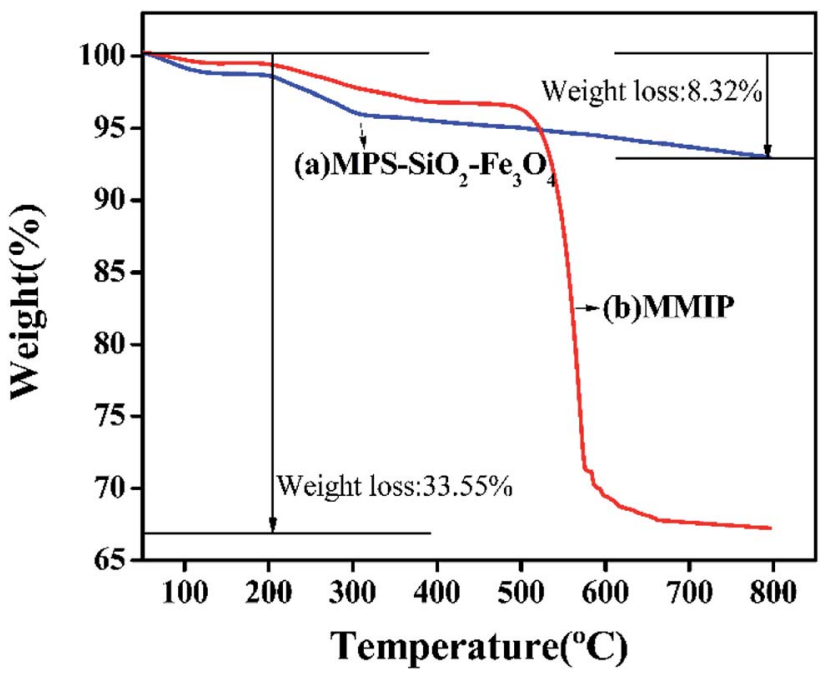

Fig. 4 Thermo gravimetric curves of MPS $-\mathrm{Fe}_{3} \mathrm{O}_{4}-\mathrm{SiO}_{2}(\mathrm{a})$ and MMIP (b). 


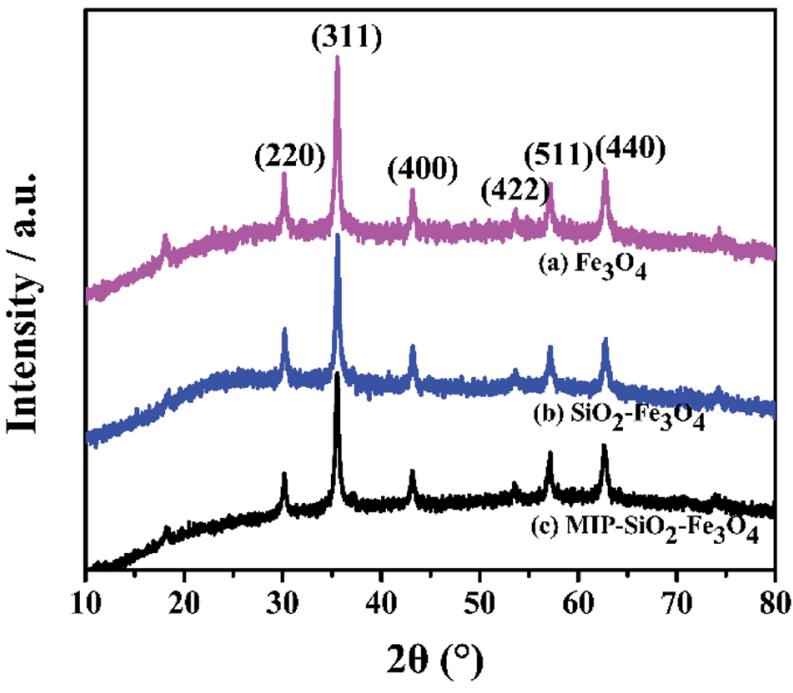

Fig. 5 XRD spectra of $\mathrm{Fe}_{3} \mathrm{O}_{4}$ (a), $\mathrm{SiO}_{2}-\mathrm{Fe}_{3} \mathrm{O}_{4}$ (b) and MMIP (c).

to $80^{\circ}$, which are indexed as (220), (311), (400), (422), (511) and (440). The peaks are assigned to the diffraction of $\mathrm{Fe}_{3} \mathrm{O}_{4}$ crystal according to the JCPDS card 19-629, and the planes indicate that $\mathrm{Fe}_{3} \mathrm{O}_{4}$ crystal is inverse spinel structure. ${ }^{23}$ However, it does not exclude the presence of $\gamma-\mathrm{Fe}_{2} \mathrm{O}_{3}$, and there may be two types of iron oxide particles of maghemite and magnetite in the synthesized sample. ${ }^{24}$ The presence of maghemite is due to oxidation of magnetite during the synthesis, ${ }^{25}$ but both iron oxide particles are magnetic and do not need to be distinguished. After modified with silica, there appears a weaker and broader peak at $2 \theta$ values from $21^{\circ}$ to $27^{\circ}$, which suggests that the amorphous silica is successfully coated on the surface of $\mathrm{Fe}_{3} \mathrm{O}_{4}$. The positions of the diffraction peaks in (a), (b) and (c) are identical, which indicates a sequence of operations do not change the crystal structure of $\mathrm{Fe}_{3} \mathrm{O}_{4}$. Compared with (a), the peak strengths of (b) and (c) are decreased significantly, indicating that the silica layer and imprinted layer have a barrier effect for magnetic $\mathrm{Fe}_{3} \mathrm{O}_{4}$.

\subsection{VSM analysis}

The magnetic properties of samples were investigated by VSM at room temperature. Fig. 6 shows the magnetic hysteresis loops of $\mathrm{Fe}_{3} \mathrm{O}_{4}, \mathrm{SiO}_{2}-\mathrm{Fe}_{3} \mathrm{O}_{4}$ and MMIP. It is apparent that three curves have similar general shape. There is no hysteresis, and the remanence and coercivity are both zero, suggesting that the samples are superparamagnetic. The saturation magnetization values of $\mathrm{Fe}_{3} \mathrm{O}_{4}, \mathrm{SiO}_{2}-\mathrm{Fe}_{3} \mathrm{O}_{4}$ and MMIP are 72.93, 60.07, 43.97 emu $\mathrm{g}^{-1}$, respectively. Because the silica and molecularly imprinted shells on the surface of $\mathrm{Fe}_{3} \mathrm{O}_{4}$ prevents its magnetic responsiveness, the magnetic property of MMIP is weaker than those of $\mathrm{Fe}_{3} \mathrm{O}_{4}$ and $\mathrm{SiO}_{2}-\mathrm{Fe}_{3} \mathrm{O}_{4}$. However, MMIP remains strongly magnetic property at room temperature and allows for as effective magnetic separation carrier. The dispersionagglomeration process of MMIP is also shown in Fig. 6. MMIP is dispersed homogeneously in the solution without external magnetic field. When the magnetic field is applied, the MMIP gathers at right side at around $20 \mathrm{~S}$, and the solution becomes

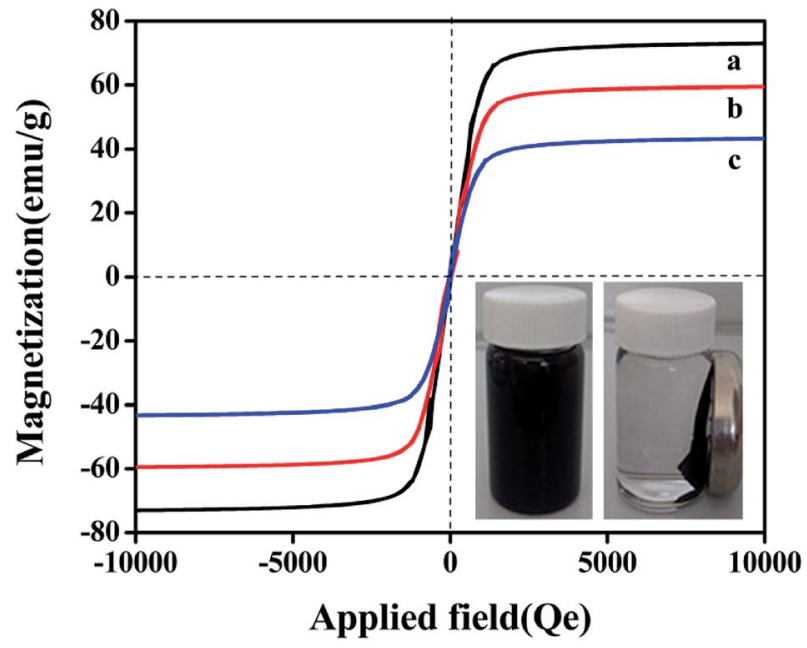

Fig. 6 Magnetization curves of $\mathrm{Fe}_{3} \mathrm{O}_{4}(\mathrm{a}), \mathrm{SiO}_{2}-\mathrm{Fe}_{3} \mathrm{O}_{4}$ (b) and MMIP (c).

clear. The above results show that the synthesized MMIP has strong magnetic properties and can realize convenient magnetic separation.

\subsection{Adsorption properties of MMIP and MNIP}

3.7.1 Static adsorption experiment. Fig. 7 shows the UV absorption spectrum of DEHP ethanol solution with different concentrations from $5 \mathrm{mg} \mathrm{L}^{-1}$ to $50 \mathrm{mg} \mathrm{L}^{-1}$. There are three absorption peaks in the curves. However, there is no red shift at the peak of $225 \mathrm{~nm}$ with the increasing of concentration, and the peak strength increases obviously. Hence, the UV absorbance of DEHP is measured at $225 \mathrm{~nm}$. The linear equation of concentration-absorbance is fitted by $y=0.0225 x-0.0118$ according to the absorbance measured at $225 \mathrm{~nm}$ with different concentrations, thereby the conversion between absorbance and concentration is obtained.

Static adsorption experiment was conducted to estimate the maximum adsorption capacity of MMIP for DEHP, and the

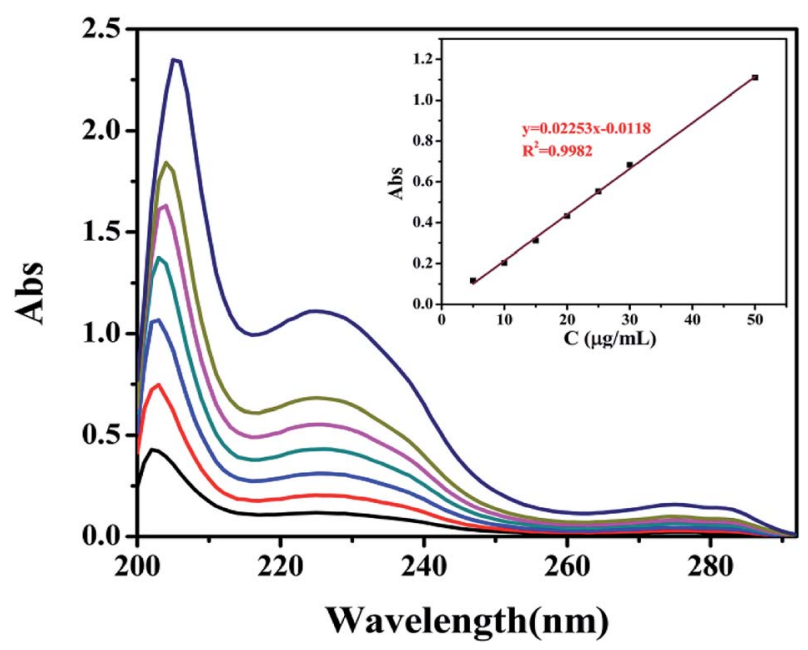

Fig. 7 UV spectra of DEHP ethanol solution with different concentrations and the linear fitting of concentration and absorbance. 


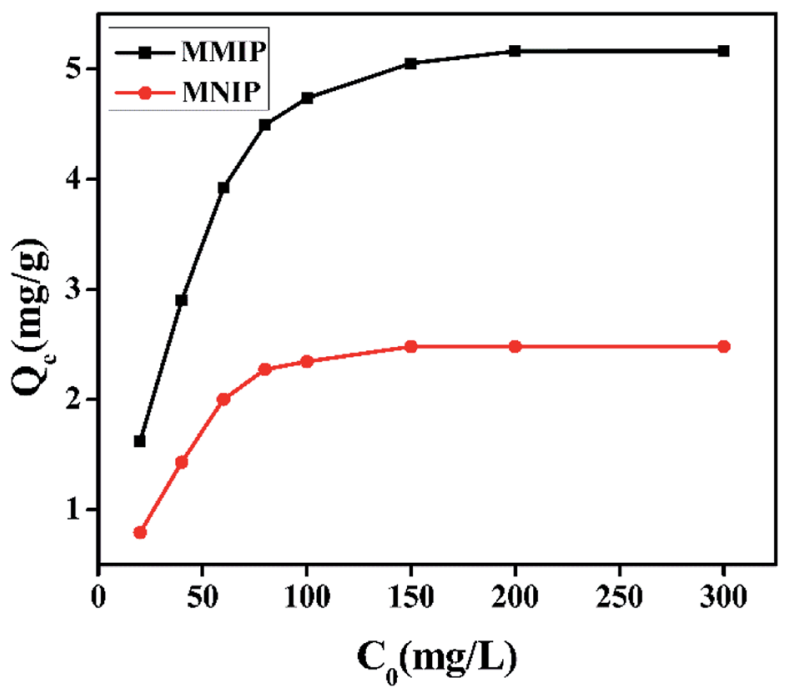

Fig. 8 The adsorption isotherms of MMIP and MNIP for DEHP.

results are displayed in Fig. 8. The binding amounts of MMIP and MNIP for DEHP increase quickly with the increasing of the initial concentration of DEHP, and at higher initial concentration over $200 \mathrm{mg} \mathrm{L}^{-1}$, the adsorption capacity becomes stable. The adsorption capacity of MMIP is higher than that of MNIP at the same concentration due to the obvious difference in the spatial structure of two polymers. The adsorption of DEHP onto MNIP is mainly dependent on the polar groups on the surface of MNIP, which is nonspecific adsorption, and the adsorption ability is relatively weak. The adsorption of DEHP onto MMIP is due to the existence of three-dimension cavities, which are complement with the spatial structure of DEHP, thus the MMIP has "memory function" to template and has strong selective adsorption ability. The difference of adsorption capacity between the two polymers is mainly due to the specific adsorption of the cavities.

Langmuir and Freundlich models are often used to describe the static adsorption process. Linear equation of Langmuir model is listed as follows:

$$
\frac{C_{\mathrm{e}}}{Q_{\mathrm{e}}}=\frac{1}{K_{\mathrm{L}} Q_{\mathrm{m}}}+\frac{C_{\mathrm{e}}}{Q_{\mathrm{m}}}
$$

where $K_{\mathrm{L}}\left(\mathrm{L} \mathrm{mg}^{-1}\right)$ is the Langmuir constant which relates to the enthalpy of sorption, $C_{\mathrm{e}}\left(\mathrm{mg} \mathrm{L}^{-1}\right)$ is the equilibrium concentration of DEHP, and $Q_{\mathrm{m}}\left(\mathrm{mg} \mathrm{g}^{-1}\right)$ is the maximum binding amount of MMIP or MNIP for DEHP.

Freundlich linear equation is described as follows:

$$
\ln Q_{\mathrm{e}}=\ln K_{\mathrm{F}}+\left(\frac{1}{n}\right) \ln C_{\mathrm{e}}
$$

where $Q_{\mathrm{e}}\left(\mathrm{mg} \mathrm{g}^{-1}\right)$ is the equilibrium binding amount of MMIP or MNIP for DEHP, and $K_{\mathrm{F}}\left(\mathrm{mg}^{1-n} \mathrm{~L}^{n} \mathrm{~g}^{-1}\right)$ and $n$ are the constants of Freundlich sorption. Table 1 shows the simulation data of MMIP and MNIP using Langmuir and Freundlich models. It is observed that the correlation coefficient of Langmuir model fitting for MMIP $\left(R^{2}=0.9997\right)$ is greater than 0.99 and much larger than that of Freundlich model $\left(R^{2}=0.9490\right)$. Langmuir fitting model is more suitable for the experimental data of MMIP, which indicates that the adsorption of template molecule on MMIP belongs to monolayer adsorption.

3.7.2 Dynamic adsorption experiment. Dynamic adsorption experiment was carried out to investigate the mass transfer properties of MMIP. Fig. 9(a) shows the adsorption kinetics curves of MMIP and MNIP for $100 \mathrm{mg} \mathrm{L}^{-1}$ DEHP ethanol solution. The adsorption capacity of MMIP for DEHP increases rapidly in the first $30 \mathrm{~min}$, then, the curve becomes relatively flat and reaches the adsorption equilibrium at about $40 \mathrm{~min}$. A similar behavior is observed for the adsorption onto MNIP. The results can be explained from the structure of MMIP and MNIP. The shallow cavities on the surface of MMIP and MNIP are favorable to the rapid adsorption for DEHP. However, when the cavities reach saturation, the process of mass transfer is subjected to resistance, and the adsorption rate decreases. Finally, the adsorption reaches adsorption equilibrium, and the adsorption capacity of MNIP increases slowly. The maximum adsorption capacity of MNIP is lower than that of MMIP owing to the absence of specific recognition sites.

Two adsorption kinetic model of pseudo-first-order kinetic model and pseudo-second-order kinetic model are employed to evaluate the dynamic binding processes of MMIP and MNIP, ${ }^{26,27}$ and the linear equation of pseudo-first-order model is expressed as:

$$
\ln \left(Q_{\mathrm{e}}-Q_{t}\right)=\ln Q_{\mathrm{e}}-k_{1} t
$$

where $k_{1}(1 / h)$ represents the pseudo-first-order kinetic rate constant, $Q_{t}\left(\mathrm{mg} \mathrm{g}^{-1}\right)$ is the binding amount of MMIP and MNIP for DEHP at contact time $t(\mathrm{~h})$.

The linear equation of pseudo-second-order model can be described as:

$$
\frac{t}{Q_{t}}=\frac{1}{k_{2} Q_{\mathrm{e}}{ }^{2}}+\frac{t}{Q_{\mathrm{e}}}
$$

where $k_{2}\left(\mathrm{~g}\left(\mathrm{mg}^{-1} \mathrm{~h}^{-1}\right)\right)$ is the pseudo-second-order kinetic rate constant, and $Q_{\mathrm{e}}\left(\mathrm{mg} \mathrm{\textrm {g } ^ { - 1 } )}\right.$ is the equilibrium adsorption capacity of MMIP and MNIP. The kinetic parameters are dis-

\begin{tabular}{|c|c|c|c|c|c|c|c|c|}
\hline & \multicolumn{4}{|c|}{ Langmuir model } & \multicolumn{4}{|c|}{ Freundlich model } \\
\hline & $K_{\mathrm{L}}$ & $Q_{\mathrm{m}}$ & $R^{2}$ & Linear fitting equation & $K_{\mathrm{F}}$ & $n_{\mathrm{F}}$ & $R^{2}$ & Linear fitting equation \\
\hline MNIP & 0.0581 & 2.6960 & 0.9929 & $y=0.3709 x+6.3796$ & 0.1866 & 1.6360 & 0.9387 & $y=0.6112 x-1.67903$ \\
\hline
\end{tabular}
played in Table 2. The results indicate that the pseudo-secondorder model yields better fitting for MMIP and MNIP between

Table 1 Langmuir and Freundlich model fitting data onto MMIP and MNIP 

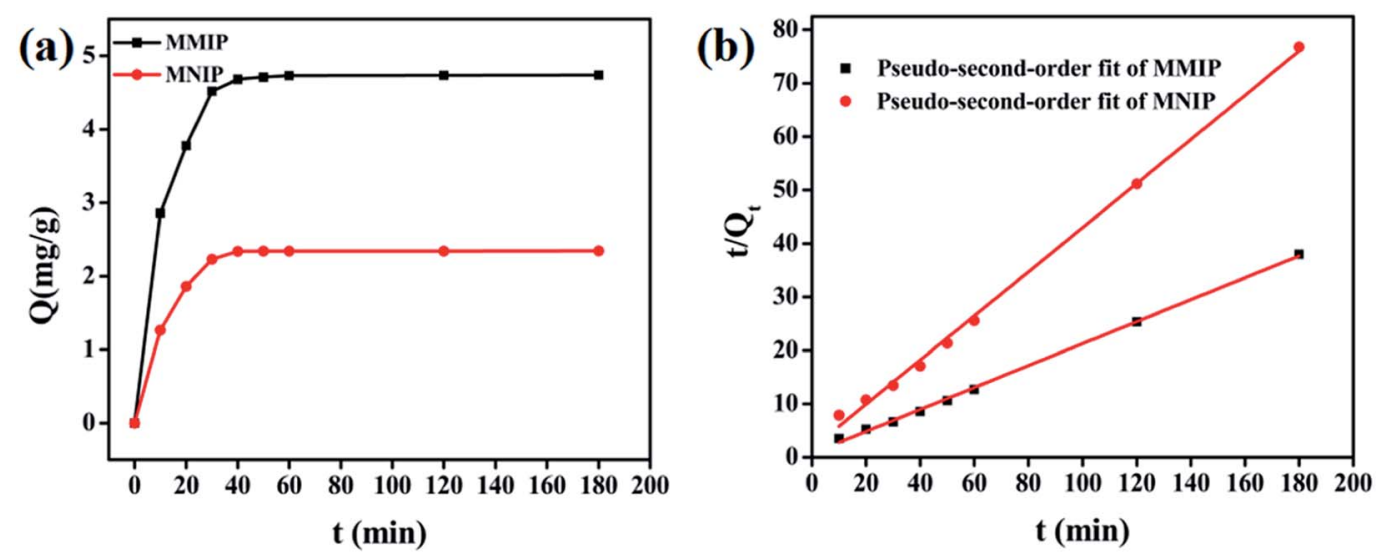

Fig. 9 Dynamic adsorption curves (a) and pseudo-second-order fitting (b) for MMIP and MNIP.

Table 2 Pseudo-first-order and pseudo-second-order kinetic model fitting data for MMIP and MNIP

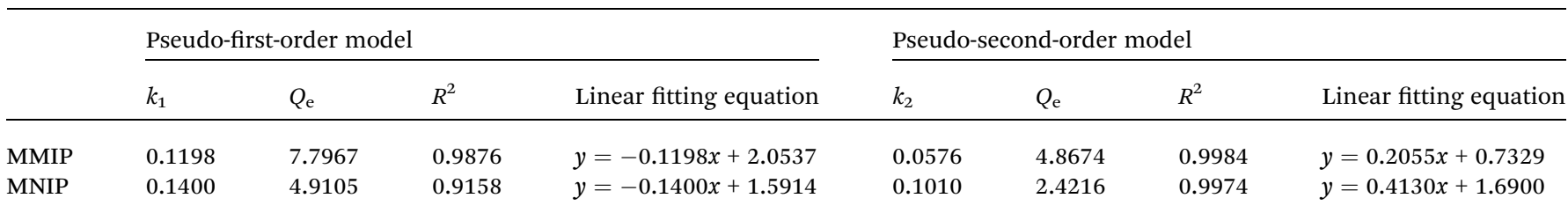

the two models with correlation coefficients of 0.9984 and 0.9974 . The maximum adsorption capacities of $4.87 \mathrm{mg} \mathrm{g}^{-1}$ for MMIP and $2.42 \mathrm{mg} \mathrm{g}^{-1}$ for MNIP calculated by pseudo-secondorder model are close to the experimental maximum adsorption amounts of $4.74 \mathrm{mg} \mathrm{g}^{-1}$ for MMIP and $2.35 \mathrm{mg} \mathrm{g}^{-1}$ for MNIP. Fig. 9(b) shows the simulations of pseudo-second-order models. The curves illuminate that the adsorption rate of MMIP for DEHP $\left(Q_{t} / t\right)$ is much larger than that of MNIP, which is due to the existence of a large number of imprinted sites on the surface of MMIP.

3.7.3 Adsorption selectivity experiment. The adsorption selectivity of MMIP for DEHP was investigated with the template structural analogues of DAP and DBP (Fig. 10) as competitive substrates. The adsorption capacities of MMIP and MNIP to the mixture of DEHP, DAP and DBP with respective concentration of $100 \mathrm{mg} \mathrm{L}$ in $2 \mathrm{~mL}$ ethanol solution are displayed in Fig. 11. The imprinting factor $\alpha$ is usually used to evaluate the recognition ability of MMIP, ${ }^{\mathbf{1 4}}$ and it is defined in the following equation: $\alpha=Q_{\mathrm{MIP}} / Q_{\mathrm{NIP}}$. The $Q_{\mathrm{MIP}}$ and $Q_{\mathrm{NIP}}$ are the adsorption capacities of template or structural analogues onto MMIP and MNIP. The imprinting factors of DEHP, DAP and DBP are 3.012,
1.547 and 1.788, respectively, which suggests that the MMIP has specific recognition performance to template molecule of DEHP. MMIP creates tailor-made binding sites with the memory of shape, size and functional groups of template molecules. ${ }^{28}$ In addition to the hydrogen bond formed between the carbonyl group in target molecules and carboxyl group embedded in MMIP, MMIP recognizing targets also depends on the shape and size. From Fig. 10, we can know DBP shares similar shape and functional groups with DEHP. However, the size of DBP is smaller, so MMIP shows lower binding capacity for DBP. The size of DAP is smaller than DBP, the binding capacity is lower than DBP. In the meanwhile, the adsorption capacities of MNIP are very close for those compounds, since there are no specific recognition sites in MNIP and the adsorption is non-selective.

\subsection{Regeneration experiment}

Reusability is an important index to evaluate the practical value of materials. The MMIP and MNIP were recycled for three times with the mixed solution of methanol and acetic acid $(v: v, 9: 1)$

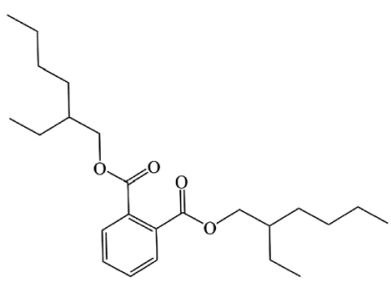

DEHP

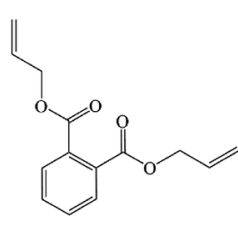

DAP

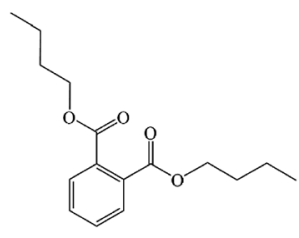

DBP

Fig. 10 Structural formula of DEHP and its structural analogues of DAP and DBP. 


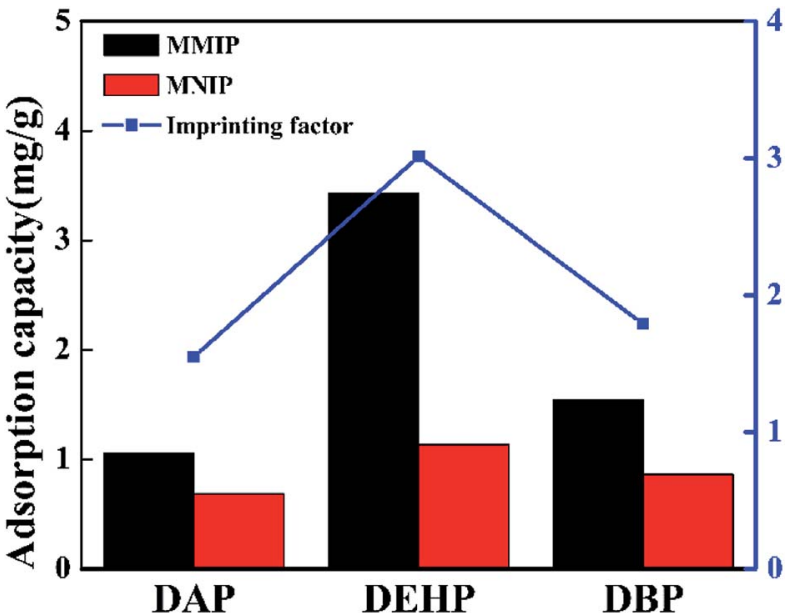

Fig. 11 Adsorption selectivities for DEHP and competing compounds onto MMIP and MNIP in the mixture solution.

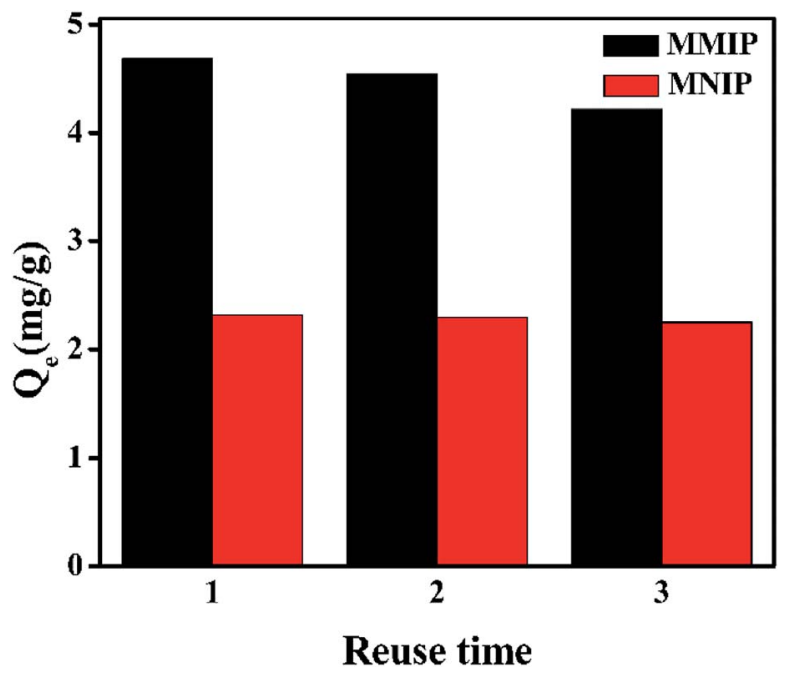

Fig. 12 Regeneration of MMIP and MNIP.

as eluent. The results are shown in Fig. 12, the adsorption capacities of DEHP on MMIP lost only $10 \%$ after three cycles, which indicates that MMIP has good and stable regeneration performance. However, the adsorption capacity of MNIP has little change. This is due to the fact that some recognition sites on the surface of MMIP are destroyed or occupied by substrate molecules during the repeated elution. Instead, MNIP is relied on nonspecific adsorption in the absence of specific recognition sites.

\section{Conclusions}

In this paper, a novel MMIP was successfully fabricated with double core-shell structure for convenient and efficient separation of DEHP from the solution by external magnetic field. The adsorption properties of MMIP and MNIP were compared by a series of static and dynamic adsorption experiments, and the adsorption selectivity of MMIP was investigated. The adsorption capacity of MMIP is always higher than that of MNIP at the same conditions. Also, Langmuir model is more suitable for the static adsorption of MMIP, which belongs to the monolayer adsorption, and the dynamic adsorption process fits the pseudo-second-order kinetic model well. Compared with DAP and DBP, the adsorption capacity of DEHP on MMIP is higher. The imprinting factors of DEHP, DAP and DBP are 3.012, 1.547 and 1.788, respectively, which indicates that MMIP has good adsorption selectivity for template molecules. The regeneration experiment shows that the adsorption capacity of MMIP has little change after three cycles, which illuminates that MMIP has good application prospect for convenient separation for DEHP.

\section{Conflicts of interest}

There are no conflicts to declare.

\section{Acknowledgements}

The work was supported by National Natural Science Foundation of China (No. 20207003, No. 20704019, No. 51603093), innovative and entrepreneurial building team project of Jiangsu Province (No. 2015026). The authors wish to express their appreciation to the Analytical Center at Jiangsu University for the measurements of samples.

\section{References}

1 J. A. Hoppin, R. Ulmer and S. J. London, Phthalate exposure and pulmonary function, Environ. Health Perspect., 2004, 112, 571-574.

2 S. H. Swan, Environmental phthalate exposure in relation to reproductive outcomes and other health endpoints in humans, Environ. Res., 2008, 108, 177-184.

3 T. Schettler, Human exposure to phthalates via consumer products, Int. J. Androl., 2006, 29, 134-139.

4 J. H. Kim, S. H. Kim, C. H. Lee, J. W. Nah and A. Hahn, DEHP migration behavior from excessively plasticized PVC sheets, Bull. Korean Chem. Soc., 2003, 24(3), 345-349.

5 J. A. Tickner, T. Schettler, T. Guidotti, M. Mccally and M. M. Rossi, Health risks posed by use of di-2-ethylhexyl phthalate (DEHP) in PVC medical devices: a critical review, Am. J. Ind. Med., 2001, 39, 100-111.

$6 \mathrm{~K}$. Haupt, Molecularly imprinted polymers in analytical chemistry, Analyst, 2001, 126, 747-756.

7 C. He, Y. Long, J. K. Pan and F. Liu, Application of molecularly imprinted polymers to solid-phase extraction of analytes from real samples, J. Biochem. Biophys. Methods, 2007, 70, 133-150.

8 F. G. Tamayo, E. Turiel and A. Martín-Esteban, Molecularly imprinted polymers for solid-phase extraction and solidphase microextraction: recent developments and future trends, J. Chromatogr. A, 2007, 1152, 32-40.

9 T. Alizadeh, M. Zare, M. R. Ganjali, P. Norouzi and B. Tawana, A new molecularly imprinted polymer (MIP)based electrochemical sensor for monitoring 2,4,6- 
trinitrotoluene (TNT) in natural waters and soil samples, Biosens. Bioelectron., 2010, 25, 1166-1172.

10 C. Malitesta, E. Mazzotta, R. A. Picca, A. Poma, I. Chianella and S. A. Piletsky, MIP sensors-the electrochemical approach, Anal. Bioanal. Chem., 2012, 402, 1827-1846.

11 M. Resmini, Molecularly imprinted polymers as biomimetic catalysts, Anal. Bioanal. Chem., 2012, 402, 3021-3026.

12 G. Wulff, Enzyme-like catalysis by molecularly imprinted polymers, Chem. Rev., 2002, 102, 1-27.

13 T. Jing, H. R. Du, Q. Dai, H. Xia, J. W. Niu, Q. L. Hao, S. R. Mei and Y. K. Zhou, Magnetic molecularly imprinted nanoparticles for recognition of lysozyme, Biosens. Bioelectron., 2010, 26, 301-306.

14 F. Ning, H. Peng, L. Dong, Z. Zhang, J. Li, L. Chen and H. Xiong, Preparation and characterization of superparamagnetic molecularly imprinted polymers for selective adsorption and separation of vanillin in food samples, J. Agric. Food Chem., 2014, 62, 11138-11145.

15 R. X. Gao, X. Kong, X. Wang, X. W. He, L. X. Chen and Y. K. Zhang, Preparation and characterization of uniformly sized molecularly imprinted polymers functionalized with core-shell magnetic nanoparticles for the recognition and enrichment of protein, J. Mater. Chem., 2011, 21, 1786317871.

16 M. L. Ma, Q. Y. Zhang, J. B. Dou, H. P. Zhang, D. Z. Yin, G. W. Chang and Y. Y. Zhou, Fabrication of onedimensional $\mathrm{Fe}_{3} \mathrm{O}_{4} / \mathrm{P}(\mathrm{GMA}-\mathrm{DVB})$ nanochains by magneticfield-induced precipitation polymerization, $J$. Colloid Interface Sci., 2012, 374, 339-344.

17 K. Haupt and K. Mosbach, Molecularly imprinted polymers and their use in biomimetic sensors, Chem. Rev., 2000, 100, 2495-2504.

18 S. R. Carter and S. Rimmer, Surface molecularly imprinted polymer core-shell particles, Adv. Funct. Mater., 2004, 14, 553-561.
19 Y. G. Cai and X. Q. Li, Effect of ammonia on the $\mathrm{SiO}_{2}$ colloidal particles synthesized by the stöber method, Appl. Mech. Mater., 2017, 865, 25-29.

20 Q. Chang and $\mathrm{H}$. Tang, Immobilization of horseradish peroxidase on $\mathrm{NH}_{2}$-modified magnetic $\mathrm{Fe}_{3} \mathrm{O}_{4} / \mathrm{SiO}_{2}$ particles and its application in removal of 2,4-dichlorophenol, Molecules, 2014, 19, 15768-15782.

21 J. Y. Ying, S. T. Selvan, D. K. Yi and S. S. Lee, Coated composites of magnetic material and quantum dots, US. pat. 7534489, 2009.

22 P. Y. Wang, X. X. Wang, S. J. Yu, Y. D. Zou, J. Wang, Z. S. Chen, N. S. Alharbi, A. Alsaedi, T. Hayat, Y. T. Chen and $\mathrm{X}$. K. Wang, Silica coated $\mathrm{Fe}_{3} \mathrm{O}_{4}$, magnetic nanospheres for high removal of organic pollutants from wastewater, Chem. Eng. J., 2016, 306, 280-288.

23 Q. L. Tao, Y. Li, Y. Shi, R. J. Liu and Y. W. Zhang, Application of Molecular Imprinted Magnetic $\mathrm{Fe}_{3} \mathrm{O}_{4} @ \mathrm{SiO}_{2}$ Nanoparticles for Selective Immobilization of Cellulase, $J$. Nanosci. Nanotechnol., 2016, 16, 6055-6060.

24 Y. Lu, Y. D. Yin, B. T. Mayers and Y. N. Xia, Modifying the surface properties of superparamagnetic iron oxide nanoparticles through a sol-gel approach, Nano Lett., 2002, 2, 183-186.

25 X. Wang, L. Wang, X. He, Y. Zhang and L. Chen, A molecularly imprinted polymer-coated nanocomposite of magnetic nanoparticles for estrone recognition, Talanta, 2009, 78, 327-332.

26 S. Azizian, Kinetic models of sorption: a theoretical analysis, J. Colloid Interface Sci., 2004, 276, 47-52.

27 I. A. W. Tan and B. H. Hameed, Adsorption isotherms, kinetics, thermodynamics and desorption studies of basic dye on activated carbon derived from oil palm empty fruit bunch, J. Appl. Sci., 2010, 10, 2565-2571.

$28 \mathrm{H}$. $\mathrm{Lu}$ and $\mathrm{S} . \mathrm{Xu}$, Mesoporous structured estrone imprinted $\mathrm{Fe}_{3} \mathrm{O}_{4} @ \mathrm{SiO}_{2} @ \mathrm{mSiO}_{2}$ for highly sensitive and selective detection of estrogens from water samples by HPLC, Talanta, 2015, 144, 303-311. 